\title{
La promoción de los derechos del niño: un discurso sin adversarios
}

\section{The promotion of children's rights: a speech without opponents}

\author{
Philippe de Dinechin ${ }^{\star}$ \\ Los derechos humanos por si mismo no son nada, \\ si no los asociamos a unas estructuras políticas \\ que son la únicas susceptibles de darles un \\ sentido. \\ Marcel Gauchet
}

\section{Resumen}

La promoción abrumante de los derechos del niño proviene de algunas (pocas) organizaciones poderosas y jerárquicas. Los expertos, altamente especializados, encuentran en Internet un medio extraordinario de difusión de sus ideas, Internet, en este caso, no es un medio que sustente el debate. La producción y sobretodo la difusión de ideas críticas son casi inexistentes, la interactividad es casi nula.La unanimidad acerca de los derechos del niño parece sospechosa. No se cuestiona aquí el objetivo perseguido: "el bienestar de los niños", sino la construcción de un sistema de pensamiento que deja muchas preguntas al margen.Este "discurso sin adversarios" no está expuesto a la crítica. Esto no significa que las críticas no son pertinentes pero que no hay actores sociales interesados por esta crítica.

Palabra clave: Derecho. Derecho del niño. Convención de Nueva York. Derechos Humanos. America Latina. Comunicación.

Doutor em Direito pela Université de Paris 3. Investigador en la Fundación Comparte, Barcelona. Barcelona - Espanha. Email: pdinechin@ hotmail.com 


\section{Abstract}

The overwhelming promotion of children's rights comes from some (few) powerful and hierarchical organizations. Experts highly specialized Internet are an extraordinary means of disseminating their ideas, Internet, in this case, is not a means to underpin the debate. Production and dissemination especially critical ideas are almost nonexistent, interactivity is almost nula.La unanimity on child rights it seems suspicious. There is no question here the objective pursued: "the welfare of children", but the construction of a system of thought that leaves many questions margen.Este "speech without adversaries" is not open to criticism. This does not mean that criticisms are not relevant but that no stakeholders concerned by this review.

Keywords: Law. Right of the child. New York Convention. Human rights. Latin America. Communication.

\section{Introdução}

Reinterpretar la Convención sobre los Derechos del Niño (CIDN) es un ejercicio arriesgado. Es también un ejercicio solitario, porque ¿quién se atreve a criticar hoy una Convención que se votó por unanimidad?

Les proponemos un ejercicio. Busquen en Internet artículos o posturas que desarrollan una visión crítica de estos derechos y de esta Convención. Los hay, pero escondidos. No aparecerán rápidamente en vuestro motor de búsqueda favorito.

Lo que aparece primero es una promoción abrumante de los derechos del niño. Las fuentes de información son homogéneas y provienen de algunas (pocas) organizaciones poderosas y jerárquicas. Los expertos, altamente especializados, encuentran en Internet un medio extraordinario de difusión de sus ideas, Internet, en este caso, no es un medio que sustente el debate. La producción y sobretodo la difusión de ideas críticas son casi inexistentes, la interactividad es casi nula.

La unanimidad acerca de los derechos del niño parece sospechosa. No se cuestiona aquí el objetivo perseguido: "el bienestar de los niños", 
sino la construcción de un sistema de pensamiento que deja muchas preguntas al margen.

Un discurso sin adversarios ${ }^{1}$ no esta expuesto a la crítica. Esto no significa que, lo analizaremos, las críticas no son pertinentes pero que no hay actores sociales interesados por esta critica.

Unas voces de unos niños chilenos legitiman este cuestionamiento². ¿De qué me sirve el derecho a la educación si no tengo una escuela pública en mi barrio? ¿De qué me sirve el derecho a la salud, cuando mi hermana murió por el sarampión? Una madre argentina nos preguntó: "De qué derechos me hablas cuando nosotras tenemos miedo de nuestros hijos ${ }^{3 "}$.

Pregunta tras pregunta nos cuestionamos: ¿Qué país puede hoy garantizar que sus niños serán educados en un "espíritu de paz, dignidad, tolerancia, libertad, igualdad y solidaridad"?, retomando los términos de las Naciones Unidas, ¿Qué jurista puede asegurar que los múltiples instrumentos de protección elaborados en materia de derechos del niño los protegen realmente?

La lenta construcción de la Convención de los Derechos del Niño se vuelve una cortina de humo destinada a esconder una realidad dura y violenta.

Paradójicamente, existe en América latina un arsenal legislativo para proteger a los niños de estos abusos, pero ¿de qué sirve?

De hecho, de las cincuenta ciudades mas violentas del mundo, 43 se ubican en América latina, 19 en Brasil, según un estudio del Consejo Ciudadano para la Seguridad Pública y la Justicia Penal de Mexico $(2014)^{4}$.

Philippe Juhem, La légitimation de la cause humanitaire : un discours sans adversaires in Revue ,Mots Année 2001 Volume 65 Numéro 1 pp. 9-27. Juhem desarrolla su hipótesis a partir del discurso de las ONGs humanitarias.

2 Talleres sobre derechos del niño, proyecto Educapaz (2005-2013) https://www.comparte.org/ category/educapaz/.

Entrevista, Tucuma, marzo 2006.

4 http://www.seguridadjusticiaypaz.org.mx/ 
A nivel mundial, la región de América Latina y el Caribe tienen la proporción más alta de víctimas de homicidio de menores de 25 años ${ }^{5}$. El mayor número de víctimas jóvenes de homicidio se registra también en Nigeria, seguido por el Brasil con aproximadamente unas 11.000 muertes.

Para Unicef, la más trágica de las violaciones de derechos que afectan a niños y niñas brasileros son los homicidios de adolescentes. De 1990 a 2013, pasó de 5 mil a 10,5 mil casos al año (Datasus, 2013), un aumento del $110 \%$. Eso significa que, en 2013, cada día 28 niños y adolescentes eran asesinados. De los adolescentes que mueren por causas externas en el país, el 36,5\% son asesinados. En la población total, ese porcentaje es del 4,8\% (Homicidios en la Adolescencia en Brasil, 2015).

1990 marca la fecha de nacimiento del Estatuto del Nino y del adolescente en Brasil, la "ley mas avanzada en el mundo en la protección de los niños", según la comisión interamericana de los derechos humanos ${ }^{6}$.

Aquí está la primera paradoja. La calidad de la legislación no implica por si sola más calidad de vida para los niños. Podríamos entonces concluir, "si tenemos una legislación más o menos adecuada, entonces el problema actual es aplicarla”. Esta conclusión viene del sentido común. pero ¿son aplicables estos derechos?

Al repasar, brevemente, algunas críticas que provienen de diversos campos de estudio nos damos cuenta que el edificio jurídico es mas frágil de lo que parece.

\section{Critica marxista}

Empezaré por una crítica clásica que se realizó hace ya casi un siglo.

\footnotetext{
5 Informe Unicef, ocultos a plena luz, 2014 http://www.unicef.es/sites/www.unicef.es/files/ informeocultosbajolaluz_0.pdf (resumen), http://files.unicef.org/publications/files/Hidden_in_ plain_sight_statistical_analysis_EN_3_Sept_2014.pdf (informe completo en ingles).

6 OEA/Ser.L/V/II.97 Doc. 29 rev.1, 29 septembre 1997
} 
Un famoso filósofo alemán escribió que los Derechos Humanos, tal cual fueron establecidos, están "al servicio de la burguesía”". Para él, se trata de derechos que formalmente pertenecen a todos los hombres, pero que realmente están reservados para los poderosos. Este aporte, en el contexto latinoamericano, no carece de picardía sabiendo que los defensores de los derechos humanos contra las dictaduras de los años setenta, recibieron ampliamente la influencia de tal filósofo. Sin embargo, una segunda lectura de esta crítica nos hace intuir que la producción exponencial de estos derechos específicos son una válvula de justicia para los niños en un mundo que, de hecho, los maltrata o los instrumentaliza.

Si se estudia el pliego de las violaciones flagrantes y masivas de los derechos del niño, nos daremos cuenta que se superpone al plano de división de las riquezas en el mundo. En términos caricaturizados, esto introduce la idea de que el discurso sobre los derechos del niño resulta ser una justificación intelectual de la violación de estos derechos. La igualdad en derecho, ¿ocultaría la iniquidad en los hechos?

El problema que se plantea entonces es cuestionarse si la CIDN, que se dirige a todos los niños y a las niñas del mundo, reduce realmente esta brecha o por el contrario, induce a un discurso engañador en materia de infancia.

En otros términos, Marx no vio en la promoción de los derechos una herramienta útil para cambiar la realidad.

\section{La concepción "universal” de los derechos.}

Marx criticó en su tiempo la noción seudo universal de los derechos. Resulta interesante cuestionar hoy dicha universalidad: es el debate del relativismo cultural.

Karl Marx, La cuestión judía, 1843, 10-18, p. 37 a 39. 
La influencia determinante de los países occidentales en la elaboración de la CIDN fue preponderante ${ }^{8}$.

Para algunas personas, esa concepción conduce a una "infantilización de los países del sur", sencillamente porque han absorbido un discurso que no han contribuido a construir.

Asimismo, el tema del relativismo cultural ha estado prácticamente ausente de los trabajos preliminares de la CIDN. Sin embargo, lineamientos culturales importantes distinguen en América Latina al niño indígena del niño de la Convención. Para las culturas indígenas la importancia del grupo prevalece sobre la realización individual, el problema de la edad del niño no tiene la misma importancia. Los problemas atinentes a la educación o a la salud responden a concepciones particulares y a veces irreductibles, dependiendo de las culturas implicadas. ¿Cuál puede ser, por ejemplo, para la cultura indígena, el alcance del derecho a la educación cuando se sabe que las nociones de niño y de educación revisten un significado propio? ¿Qué sucede hoy en día con los niños latinoamericanos cuando tienen acceso a derechos pensados a partir de la realidad de la infancia occidental?

La simultaneidad y la homogeneidad de las reformas que han acompañado a La CIDN permiten plantearse el interrogante sobre "la mano invisible" que los guía. Occidente, más que "querer" transmitir explícitamente una visión de la infancia, transmite ideas y conceptos que uno encuentra tanto en el área cultural como jurídica ${ }^{10}$. Marcan lo imaginario colectivo e influyen en la vida cotidiana. De hecho, el "American way of life" es un sueño para muchos jóvenes latinoamericanos. De este modo, progresivamente, se impone una visión particular del niño fuertemente impregnada por la corriente anglosajona "liberal".

Deler, Fauré, Piveteau, Roca (1988), p. 23. Ver igualmente Pilotti (2001), p. 13.

V. Pupavac, "The infantilisation of the South and the UN Conventiion on the rights of the Children" in Student Human Rights Law Centre, Newsletter, marzo de 1998, p.3.

10 Ver los trabajos de Dezalay y Garth, La internacionalización de las luchas por el poder, Editorial Universidad Bolivariana, 2002. 
En términos generales, limitarse a exigir la aplicación de la CIDN de manera profética ${ }^{11}$ o angelical ${ }^{12}$ sin leer las "estructuras" que la acompañan es más que un riesgo de mala interpretación jurídica, es un error en cuanto a la eficacia del fin perseguido y compartido: el bienestar de la infancia.

\section{Derechos y frustración}

La filosofía del derecho nos enseña un limite hasta ahora poco cuestionado del alcance de los derechos humanos. Mencionamos sin mayor análisis la pertinencia de Michel Villey, en su obra "El derecho y los derechos humanos ${ }^{13}$ ". Para el filósofo, los derechos humanos, originados en la Europa moderna, pertenecen al campo de la moral y no del derecho. Al criticar los tratados en materia de Derechos Humanos, Villey señala que se trata de aspiraciones y no de derechos verdaderos. Hay una confusión entre el derecho y sus fines.

El filosofo señala que esta "superabundancia" (de derechos humanos) sirve sobretodo para mantener un mar de pretensiones imposibles de apaciguar, que cuando caen sobre la realidad hacen que las personas se decepcionen y se amarguen. Cuando estas "personas" son niños, la amargura es aún más grande. Para él, los derechos son ilusorios, contradictorios entre si e inaplicables. Conceder derechos a algunos grupos de la población puede conllevar a grandes injusticias, algunas veces peores que aquellas que se querían combatir. $Y$ estos derechos subjetivos crean una ilusión. Es la ilusión de que todo es posible para todo el mundo, en nombre de de los derechos humanos.

La hipótesis de la frustración, elaborada por Michel Villey, se concreta hoy, por ejemplo, en la visión de que el niño sabe que tiene sus

\footnotetext{
11 E. García Méndez, "La dimensión política de la responsabilidad penal de los adolescentes de América Latina, notas para la construcción de una modesta utopía", in Justicia y derechos del niño, $n^{\circ} 3$, Santiago, diciembre de 2001, p. 85 a p.102.

12 ONG que trabajan con la infancia.

13 Villey, M. Le droit et les droits de l'homme, PUF, Paris, 1990.
} 
derechos. Durante el Foro de la Infancia con el auspicio de las Naciones Unidas en el año 2002 más de cuatrocientos niños de ciento cincuenta y dos países proclamaron un texto ante sesenta Jefes de Estado, dando conocimiento de sus frustraciones y de sus deseos. Ellos declararon: “... Nosotros somos los niños cuyas voces no se oyen: es hora de que nos tomen en cuenta ${ }^{14}$...". Haciéndose eco del discurso "oficial" sobre la infancia, el entonces Secretario General de las Naciones Unidas, Kofi Annan, dijo: "Estos derechos les corresponden indiscutiblemente. Y sin embargo, nosotros, los adultos, os hemos defraudado deplorablemente y faltado a nuestro deber de defenderles a todos". Pálido reflejo de las frustraciones reales de los niños y niñas cuyos derechos son burlados, el texto propuesto por los niños presentes en la sesión concluyó con la promesa de que ellos defenderán los derechos de la infancia cuando sean adultos. Como por un "juego de espejos", las promesas de los niños responden a las promesas de los adultos dándole al derecho el carácter ilusorio evocado por Villey.

Incapaces de cambiar substancialmente la realidad, los derechos de la infancia son una promesa siempre para el futuro.

\section{Los derechos enredados}

Otra crítica nos viene de la sociología del derecho. Dice el jurista sociólogo Carbonnier que el movimiento de desarrollo de los derechos subjetivos, que incluyen los derechos del niño, es doble: ellos se imponen y ellos se ramifican ${ }^{15}$. Las posibilidades de actuar para los grupos provistos de derechos subjetivos se incrementan y se distinguen.

Como en una especie de partenogénesis, los derechos se multiplican tan pronto nacen y se reproducen a partir de ellos mismos.

\footnotetext{
14 "Un mundo apropiado para nosotros", Foro de la Infancia, New York, 5-7 de mayo de 2002, Reunión de delegados de menores de 18 años de edad en la Sesión Extraordinaria de las Naciones Unidas consagrada a la infancia.

15 Carbonnier, J. Droit et passion du droit sous la cinquième république, Forum Flammarion, Paris, 1996. p.121 a 126.
} 
El asunto se complica cuando el sujeto de estos derechos forma parte de varios grupos titulares de derechos subjetivos. Asimismo, una joven niña guaraní del Paraguay podría hacer prevalecer sus derechos como niña, como mujer, como joven y como indígena, según los intereses que estén en juego.

La consecuencia más amenazadora, para el sociólogo francés, es un retroceso de los valores colectivos fundadores del Estado de derecho.

Es con este espíritu que la comunidad de ciudadanos, la República para Carbonnier, está en retroceso, porque existen muchas oportunidades de que el bien del grupo (niño, mujer, joven o indígena) entre en conflicto con el bien común y probablemente con el bien de otros grupos también legítimos (sin entrar en las distinciones entre derechos del niño y derechos de la niña, derechos de los adolescentes y derechos de los jóvenes).

De este modo, los derechos del niño se enfrentan a otra contradicción. La única forma de tender hacia la efectividad de estos derechos es hacerlos más precisos. Sin embargo, la precisión implica una complejidad a veces tan grande que tiene como resultado la inaplicabilidad. La escasez de derechos es más fácil de administrar que la abundancia.

En dos décadas, más de un centenar de documentos de alcance internacional han aparecido en esta área, sin que se sepa con precisión que importancia tiene un tratado en relación a otro y sin una preocupación clara sobre la articulación de estas disposiciones entre sí.

Por la complejidad del derecho, la multiplicación de las normas y de las instancias, se asiste entonces al debilitamiento del Estado de derecho.

A todo esto hay que añadir un elemento de debate relativamente nuevo: la mutación del Estado de derecho en la "sociedad de derechos". En el momento en el que el esfuerzo por la coherencia jurídica prácticamente es coronado por el éxito, el eje a partir del 
que se desarrolla (el Estado) se descentra. En los hechos, el Estado no es el único actor responsable de satisfacer los derechos, aunque se le exija que sea el principal responsable de su realización. Jean Michel Blanquer ${ }^{16}$ considera que la noción puede ser definida "como una instrumentalización por parte de personas morales y físicas, de los mecanismos y contenidos de los diferentes niveles de derecho (...) en el marco de estrategias específicas de defensa de sus intereses". Así pues, un individuo puede elegir según sus intereses y según la oferta de derechos.

De facto, la multiplicidad de las normas y de las estrategias conduce a un dominio del derecho y del discurso sobre el derecho en el plano del debate político. Los recientes debates sobre la responsabilidad penal de los menores son un ejemplo de ello.

El niño nunca había sido tan estudiado, y sin embargo nunca había parecido tan inasible. Se incrementa la diferencia entre el niño protegido de la clase media y el niño trabajador de las clases desfavorecidas. Por ello coexisten infancias difíciles de reunificar para el derecho.

La preocupación de principios de siglo por las desgracias que sufría el niño se ha transformado hoy en un debate pasional. La pasión actual por el niño no es más que la traducción de una inquietud que acompaña a la globalización. Sin recursos operativos para comprender el mundo, el niño se convierte en el refugio de lo proyectivo. Del mismo modo, el derecho se proyecta, se distribuye y se subjetiviza.

Decir que los niños tienen derechos se traduce jurídicamente en un mayor control del Estado sobre ellos. Se perfila un nuevo paternalismo de Estado, omnipresente en la Convención, que corre el riesgo de producir una falta de inversión de los padres en el cuidado de los hijos ${ }^{17}$ y un debilitamiento del derecho. El debate sobre la función

16 J. M. Blanquer, Entre "Etat de Droit" et "société de droit": l'Amérique Latine à la recherche d'un concept directeur, en Forum développement et culture, París: Unesco, 11 y 12 de marzo de 1999, T 4, p. 10.

17 La multiplicación del número de juicios de niños contra sus padres resulta un claro ejemplo de este fenómeno. 
del derecho tiene lugar en un contexto en el que las ideas liberales se han impuesto, es decir, la redefinición del derecho, tan anhelada por la doctrina de la protección integral, se ha efectuado al mismo tiempo que una retirada progresiva del Estado protector. Este tema no ha sido abordado por los doctrinarios, lo que se traduce sin embargo para la infancia en una disminución de las políticas públicas y en la emergencia de nuevos actores que intentan reemplazarlos ${ }^{18}$. La apuesta por un derecho utópico se produjo al mismo tiempo que el debilitamiento del Estado que se suponía que debía implantarlo.

En este contexto, la CIDN conlleva una nueva paradoja. En el texto internacional, el Estado es un actor clave en el despliegue y en el cumplimiento de los derechos del niño. Ahora bien, con el advenimiento del liberalismo en el continente, el Estado se ha ido retirando progresivamente de numerosos sectores. Esta disminución del sector público tiene consecuencias directas en la infancia y contribuye a animar la paradoja: ¿cómo exigir al Estado una protección mayor a favor de la infancia mientras que sus medios de acción para hacerlo son, por voluntad política, reducidos significativamente?

Los temores expresados por UNICEF desde $1987^{19}$ ante el aplazamiento de los planes sociales por los proveedores de fondos y ante el riesgo de una disminución de los gastos público estaban justificados. El Estado, en América Latina, se retiró. Un gran número de servicios públicos fue privatizado. La educación y la salud son privilegios que ahora comparten el sector público y el privado. En este debate, el Estado es el juez, aunque ya no controle las reglas del juego.

La sociedad de derechos ${ }^{20}$ es democráticamente seductora, da un amplio lugar a la participación de los ciudadanos organizados. Los

\footnotetext{
18 N.Affre, "ONG et régulation étatique en Amérique Centrale », en Deler, Fauré, Piveteau, Roca (1998), p. 651. Natalie Affre, doctora en Ciencias Políticas, lo ejemplifica de la siguiente forma: "En los países latinoamericanos, de forma particular, la tendencia al crecimiento del sector de las ONG y a la retirada del sector público se ha visto muy acentuada por la difusión de las ideas neoliberales".

19 G. Cornia, R. Jolly, F. Stewart, L'Ajustement à visage humain: protéger les groupes vulnérables et favoriser la croissance, París: UNICEF Economica, 1987.

20 J.M. Blanquer, "Consolidación democrática? Para un enfoque constitucional", Revista Pouvoirs, $n^{\circ}$ 98, 2001, p.46.
} 
ejemplos son legión en el área de las normas relativas a la infancia en donde la participación está muchas veces integrada en la ley. Por otra parte, la multiplicación de las instancias reguladoras de los derechos del niño contribuye a la confusión. En cambio, ella sustituye algunas veces un principio unificador, responsable, único de la efectividad de los derechos. ¿Qué hay qué esperar por tanto de esta tendencia al pluralismo, cada vez más marcada en lo que atañe al derecho de la infancia? Los logros, en términos de participación en la elaboración y en la implementación de la norma, se hacen algunas veces en detrimento de su eficacia. El derecho de los niños se vuelve entonces retórico en esta parte del mundo. Para un gobierno, los potenciales frutos políticos de una integración a su derecho interno de las normas de los derechos del niño son suficientemente seductores para que se lance en la promulgación de textos sin preocuparse tanto de su aplicabilidad.

Por la multiplicación de las normas, de las instancias y los procedimientos, el derecho no ha pasado simplemente a ser más complejo. Se ha vuelto enredado. Nace de este fenómeno una nivelación de los recursos posibles que socavan la coherencia del Estado de derecho, porque ya no es el amo indiscutible de la jerarquía de las normas, sino que pasa a ser el mediador discutido.

Es la victoria de un derecho difícil de delimitar. ¿Quién puede tener hoy en día una comprensión exhaustiva del derecho de la infancia? La complejidad implica, a la vez, comprensión y especialización. La categoría de experto jurídico de la infancia sale a la luz. Los jueces y los abogados también se especializan. En las facultades, los cursos de derecho sobre la familia se separan para darle un lugar más importante a los derechos del niño. La declinación de los derechos humanos en derechos específicos pronto es seguida por la diversidad de los productos universitarios. En la lógica del mercado jurídico, obras y cursos especializados en los derechos de los niños y las niñas se multiplican. Sin embargo, para los juristas la comprensión del dominio no es siempre fácil. Resulta todavía más ardua para los principales beneficiarios de estos derechos: los niños y las niñas. Esta complejidad en las normas hace que nadie domine verdaderamente el tema. "Los 
sistemas normativos contemporáneos, expresa el profesor Perrin ${ }^{21}$, se han transformado en una complejidad tal que la voluntad prácticamente no puede nunca ser verdaderamente informada o ilustrada". El niño se encuentra rodeado por una "red normativa" que se caracteriza por el "volumen de las producciones", al que se le agrega "la complejidad de las interrelaciones entre los sistemas normativos".

\section{Perspectivas}

« Observamos con crudeza el abismo existente entre el derecho de los libros y la praxis social » escribe María Luisa Bartolomei22, especialista de los derechos humanos. Sustenta su reflexión mostrando que si bien los Bolivar y los criollos se inspiraron de las declaraciones francesa y americana de derechos humanos, en realidad el cambio de las colonias a las independencias no ha cambiado nada en las estructuras sociales. Las ideas de libertad fueron, para la jurista, un instrumento para justificar el movimiento de independencia.

El disfrace jurídico es constante en la vida publica latinoamericana. En este sentido, la historia real de los derechos humanos no es la historia de los textos pero de los hechos de resistencia contra los poderes.

De allí viene el papel simbólico de derecho puesto en evidencia (tomando como ejemplo el caso colombiano) por Garcia Villegas ${ }^{23}$. Las ganancias políticas de un alineamiento de las legislaciones sobre los principios de derechos humanos son para un gobierno suficientemente seductoras para que se lance en la promulgación de texto sabiendo que su aplicación será casi imposible.

21 J.F. Perrin, "La autonomía de la voluntad y el pluralismo jurídico, hoy", in Comunicación para el XVII Congreso Internacional de Sociólogos de lengua francesa, ISLF, Tours, 2004. En su conferencia, Perrin no se refiere explícitamente a los derechos de los niños y las niñas. El ejemplo elegido es el de la medicina. La analogía con los derechos del niño es nuestra.

22 M.L.Bartolomei, " Diversidad en la conceptualización de los derechos humanos: diversidad cultural en América Latina », El otro derecho volumen 19, ILSA, Bogotá, octubre 1995.

23 M. Garcia Villegas, La eficacia simbólica del derecho. Examen de situaciones colombianas, Uniandes, Bogotá, 1993, p.75. 
Aquí tenemos una de las paradojas de los derechos del niño cuyo carácter obligatorio es, lo vimos en el caso de Brasil poco operante. Queda entonces su papel simbólico o moralista, tal vez más eficiente. De este punto de vista podemos ver que el cambio provocado por la irrupción de los derechos del niño en el continente no ha cambiado casi nada en la forma de tratarlos. Visto así, los testimonios de los juristas que convergen en el lema "si los derechos del niño sirven" reflejan buenas intenciones pero no se traduce en los hechos, en la vida real de los niños.

Estos derechos serian entonces una utopía. Hablan del niño como debería ser considerado en el continente. Este niño (con sus derechos) tendría acceso a una escuela de calidad, a una salud de calidad, tendría el derecho de organizarse... Este niño es escaso hoy en día, es el niño "rico".

Del nuevo mundo de los conquistadores al hombre nuevo de los revolucionarios, América latina fue muchas veces entendida como debería ser y no como es. La utopía - o sea el lugar de todos los posibles - del niño nuevo funciona del mismo modo. La promesa de un futuro mejor que permite aceptar un presente inaceptable.

De este punto de vista, cabe esperar que cambien las mentalidades o las practicas para que se aplique el derecho como lo sugieren varios juristas y bien multiplicar los recursos de amparo (largos, inciertos y caros) para que el derecho internacional de protección de los niños se haga efectivo.

Sin embargo, para Eugenio Raúl Zaffaroni, Juez de la Corte Suprema de Justicia argentina (2003/2014), la ley nacional tiene mecanismos e instrumentos para ponerla en funcionamiento. ${ }^{24}$

24 Philippe de Dinechin, Nihil Olivera, Los hechos se burlan del derecho, Miradas cruzadas sobre la efectividad del derecho a la educación en los barrios perifericos de San Miguel de Tucuman, Edición Deriva, febrero 2009. 
"Hay que meter amparo ${ }^{25}$ por todos lados. Yo creo que lo que podemos hacer desde acá es resolver amparo. Hay una cosa para lo que las Convenciones fundamentalmente sirven, que es dar instrumentos jurídicos. El instrumento jurídico existe y hay que usarlo".

La tarea queda pendiente para las nuevas generaciones de abogados. Sin duda, un desafío del siglo 21 .

\section{Conclusión}

Una primera conclusión podría ser que los derechos del niño estén al servicio de unos pocos, que son enredados, complicados, con una impronta occidental que, finalmente, parecen no solucionar lo que garantizan resolver.

Tanto desde la pedagogía como de la filosofía, pasando por el derecho, observamos que el Corpus Juris de los derechos del niño no es tan sólido como parece. El hecho sigue rebelde.

Estamos obligados a constatar que pese a los esfuerzos durables de la comunidad internacional, pese a las interpretaciones de diversos juristas, filósofos e intelectuales un gran número de niños permanece hoy marginados, maltratados y olvidados.

A nuestro juicio, se ha sobreexplotado el derecho y se ha dejado de lado la reconstrucción de un proyecto colectivo y político. Esta observación debe ser leída al aliso de las lógicas de poderes que sin duda son menos engañosas que los conceptos teóricos sobre la niñez, sospechosos de manipulación.

La situación actual nos recuerda el Hans Christian Andersen llamado "El traje nuevo del Emperador", donde unos costureros diseñan

25 Para Zaffaroni, frente a la vulneración del derecho a la educación se debiera utilizar la interposición de un recurso de amparo. Según el artículo 1 de la Ley 16986 (1966): “La acción de amparo será admisible contra todo acto u omisión de autoridad pública que, en forma actual o inminente, lesione, restrinja altere o amenace, con arbitrariedad o ilegalidad manifiesta los derechos o garantías explícita o implícitamente reconocidas por la Constitución Nacional, con excepción de la libertad individual tutelada por el "hábeas corpus"”. 
un traje invisible para el Emperador que sólo pueden ver las personas "inteligentes". Todos los cortesanos, ante el miedo de parecer tontos y no inteligentes, dicen que el traje del Emperador es una maravilla, hasta que un niño ajeno a tales connotaciones grita: « ¡Pero el rey anda desnudo!».

Hoy en día nos maravillamos ante "Los Derechos del Niño", como si fueran "el traje" perfecto para su protección, hecho a la medida de los juristas más inteligentes. Ahora, no es el rey quien va desnudo sino el niño que, paradójicamente, tiene más derechos para protegerlo de todos los males. ONG, UNICEF, juristas y psicólogos se empeñan en discutir sobre los derechos del niño sin atreverse a reconocer que no son tan útiles. Los derechos del niño distraen las conciencias.

Andersen acaba su cuento y nosotros el nuestro diciendo: "Nadie permitía que los demás se diesen cuenta de que no veían nada, porque eso hubiera significado que eran indignos de su cargo o de que eran tontos de remate".

\section{Referências}

DE DINECHI, Philippe. Cultivons la paix (cultivamos la paz).

Entrevista con Adolfo Perez Esquivel. Paris: Ed. DDB, 2000.

DE DINECHI, Philippe. México: la solidaridad hoy. México: Universidad de Monterrey, 2000.

. Identidades y reconversión en la ciudades mineras de

Lota y Coronel. Chile: Ed Lom, 2001

. Ser solidario hoy. Revista de Opinión y Pensamiento, [s. I.], n. 52, 2002.

. La dominación por la ayuda. Tribuna del Bio-Bio, Chile,

2004.

; BOCAZ, Monica. In: Semanas sociales de Francia: "Familia y Sociedad" (testimonio), sept. 2005. 
. In: CONFERENCIA, UNIVERSIDAD AUTÓNOMA DE

BARCELONA: "Desafíos para los derechos del niño", Barcelona, 2005. Anais... Barcelona, 2005.

. Los derechos humanos nacen a partir de los abusos. Revista Comparte, Barcelona, marzo 2006.

; OLIVERA, Nihil. La Vergüenza de la inmigración ecuatoriana en España. Barcelona, 2006.

; OLIVERA, Nihil. La inmigración ecuatoriana en España. Barcelona: Universidad Autónoma de Barcelona, 2007.

. Derechos del niño y coherencia del Estado de derecho. In: CONGRESO MUNDIAL SOBRE DERECHOS DE LA NIÑEZ Y ADOLESCENCIA, 3., 2007, Barcelona. Anais... Barcelona, 2007.

. Cultivamos la paz. Barcelona: Ed. Deriva, 2007.

; OLIVERA, Nihil. Prensa e inmigración ecuatoriana: un modelo para mitigar las contradicciones sociales. In: SIMPOSIO INTERNACIONAL - GEDIME, 2008, Barcelona. Anais... Barcelona, 2008.

; OLIVEIRA, Nihil. Los hechos se burlan del derecho:

miradas cruzadas sobre la efectividad del derecho a la Educación en los barrios periféricos de San Miguel de Tucumán, Argentina. Barcelona: Ed. Deriva, 2009.

; OLIVERA, Nihil. Immigracio, infancia, familia y suport profesional. Barcelona: Universidad de Barcelona, 2009.

. Introdución a una critica de los derechos del niño. Revista Juridica Ad Hoc, Buenos Aires, 2012.

. Introducion critica a los derechos del niño. Pensar: Revista de Ciências Jurídicas, Fortaleza, v. 20, n. 2, p. 535-580, maio/ago. 2015.

Recebido em: 02/02/2016

Aprovado em: 31/05/2016 